\title{
PULVERIZACIONES AGRÍCOLAS: COMPARACIÓN DE METODOLOGÍAS PARA SU EVALUACIÓN
}

\section{AGRICULTURAL SPRAYS: COMPARISON OF EVALUATION METHODS}

\author{
Victor Hugo Merani ${ }^{10 *}$, Facundo Guilino ${ }^{1 \mathrm{~b}}$, Mariano Ponce ${ }^{1 \mathrm{c}}$, Juan Manuel Vázquez ${ }^{1 \mathrm{~d}}$, Luciano \\ Larrieu $^{1 \mathrm{e}}$, Ana Laura Giambelluca ${ }^{1 f}$, Pedro Platz ${ }^{2 a}$, Santiago Tour ${ }^{2 b}$ y Matilde Mur ${ }^{1 \mathrm{~g}}$ \\ ${ }^{1 a}$ CISSAF. Facultad de Ciencias Agrarias y Forestales. Universidad Nacional de La Plata. Calle 60 y 119 \\ s/n - CP 1900. La Plata, Buenos Aires, Argentina \\ https://orcid.org/0000-0002-0681-7535 \\ ${ }^{1 b}$ CISSAF. Facultad de Ciencias Agrarias y Forestales. Universidad Nacional de La Plata. Calle 60 y 119 \\ s/n - CP 1900. La Plata, Buenos Aires, Argentina \\ https://orcid.org/0000-0002-2337-3833 \\ ${ }^{1 c}$ CISSAF. Facultad de Ciencias Agrarias y Forestales. Universidad Nacional de La Plata. Calle 60 y 119 \\ s/n - CP 1900. La Plata, Buenos Aires, Argentina \\ https://orcid.org/0000-0002-9917-0282 \\ 1d CISSAF. Facultad de Ciencias Agrarias y Forestales. Universidad Nacional de La Plata. Calle 60 y 119 \\ s/n - CP 1900. La Plata, Buenos Aires, Argentina \\ https://orcid.org/0000-0001-5825-3834 \\ ${ }^{1 e}$ CISSAF. Facultad de Ciencias Agrarias y Forestales. Universidad Nacional de La Plata. Calle 60 y 119 \\ s/n - CP 1900. La Plata, Buenos Aires, Argentina \\ https://orcid.org/0000-0002-9520-158X \\ ${ }_{1 f}$ CISSAF. Facultad de Ciencias Agrarias y Forestales. Universidad Nacional de La Plata. Calle 60 y 119 \\ s/n - CP 1900. La Plata, Buenos Aires, Argentina \\ https://orcid.org/0000-0002-8551-8348 \\ Ig CISSAF. Facultad de Ciencias Agrarias y Forestales. Universidad Nacional de La Plata. Calle 60 y 119 \\ s/n - CP 1900. La Plata, Buenos Aires, Argentina \\ https://orcid.org/0000-0002-6055-2638 \\ 2a Universidad Nacional de Mar del Plata. Ruta Nacional 226, Km73,5 - CP 7620. Balcarce, Buenos Aires, \\ Argentina \\ https://orcid.org/0000-0003-2454-9359 \\ ${ }^{2 b}$ Universidad Nacional de Mar del Plata. Ruta Nacional 226, Km73,5 - CP 7620. Balcarce, Buenos Aires, \\ Argentina \\ https://orcid.org/0000-0002-5977-8218 \\ * Autor para correspondencia e-mail: victor.merani@agro.unlp.edu.ar
}

\section{RESUMEN}

Se realizó un ensayo con el objetivo de comparar colectores artificiales (papel hidrosensible y papel fotográfico Glossy) y dos programas informáticos (Gotas-PC y CIR1.5) para la valoración de una aplicación con diferentes diseños de boquillas (XR11002VP y TXA8002VK). Los parámetros evaluados fueron DV0,5, densidad de impactos de las gotas y eficiencia; este último se comparó como contraste de la determinación colorimétrica del trazador azul brillante. Se trabajó dentro de un galpón cerrado, bajo condiciones semicontroladas, sin viento y sobre terreno uniforme. La densidad de impactos no presentó diferencias entre colectores para el programa Gotas-PC, pero sí para CIR1.5. Al utilizar colectores hidrosensibles, la eficiencia es sobrestimada con el programa 
CIR1.5 y subestimada con Gotas-PC. Con colectores fotográficos, ambos programas subestiman, debido a que presentan improntas más pequeñas que arrojan valores de DV0,5 y eficiencia menor. En aplicaciones con población de gotas de menor tamaño, los porcentajes de eficiencia son similares con los dos programas. El programa CIR1.5 es más sensible al momento de analizar las gotas sobre ambos colectores y es capaz de distinguir mayor número de gotas y de menor tamaño.

Palabras clave: CIR1.5, papel hidrosensible, papel fotográfico, Kromecote, Gotas-PC.

\section{ABSTRACT}

A study was conducted in order to compare two types of artificial collectors (water-sensitive paper and photographic paper) using two software programs (Gotas-PC and CIR1.5) for the assessment of spray applications with two different nozzle types (XR11002VP and TXA8002VK). The evaluated parameters were DV0.5, droplet number density and efficiency, this was compared to the colometric determination with bright blue tracer. The trial was conducted inside a closed facility, under semicontrolled conditions, in absence of wind and on a uniform level ground. Droplet number density did not show significant differences between collectors using Gotas-PC, but differences were found with CIR1.5. For water-sensitive paper, efficiency is overestimated with CIR1.5 software but underestimated with Gotas-PC. However, both software programs underestimate with photographic paper according to the smaller imprints that result in lower values of DV0,5 and efficiency. In applications with smaller droplet population, efficiency is similar with both software programs. The CIR1.5 software is more sensitive for analyzing drops on both collector types and can distinguish a larger number of drops and smaller sizes.

Keywords: CIR1.5, water-sensitive paper, photography paper, Kromecote, Gotas-PC.

\section{INTRODUCCIÓN}

El abordaje de la problemática de las plagas en los sistemas agrícolas actuales es realizado, en su mayoría, por métodos de control químico.

Argentina es el tercer país en superficie sembrada con cultivos genéticamente modificado, por detrás de EE.UU. y Brasil (ISAAA, 2017). Impulsado por estos cultivos $\mathrm{y}$, especialmente, por la siembra directa, el barbecho químico y la soja Roundup Ready (RR) han llevado a un incremento significativo en el uso del herbicida glifosato, provocando que, en poco más de 20 años, se conocieran 15 especies resistentes al mismo (Villalba, 2009). La aparición de resistencias conllevó a un aumento en el uso de otros herbicidas con el afán de mantener el control de las malezas dentro del sistema de siembra directa, lo que generó la existencia de más de 460 ingredientes activos diferentes y 5200 formulados en la actualidad (SENASA, 2020). Por otro lado, el aumento de la producción agrícola $\mathrm{y}$, especialmente, la adopción de un sistema de monocultivos, ha creado un aumento extraordinario de insectos-plagas y enfermedades especializados en estos cultivos (Czepak et al., 2013; Zarrilli, 2020).

A pesar de los beneficios obtenidos por el uso de los plaguicidas, estos productos químicos no dejan de plantear diferentes dificultades, su aplicación no es neutral en sus consecuencias para el medioambiente, tanto en las posibilidades de contaminación y la reducción de la biodiversidad, como en sus consecuencias sanitarias por la exposición a agentes químicos (Carriquiborde, 2010; Simoniello et al., 2010; Bernardos y Zaccagnini, 2011).

Las pulverizaciones hidráulicas son la forma más difundida de aplicación de fitosanitarios, las mismas son de complejo control y generan un amplio rango de tamaños de gotas, generalmente de $900 \mu \mathrm{m}$ a $<10 \mu \mathrm{m}$. El estudio de las características de la población de gotas que alcanzan el blanco, resulta imprescindible para determinar la calidad y eficiencia de la aplicación, ya que ellas son el medio que permite transportar el principio activo hasta el objetivo. El estudio de estas gotas es complejo dado la variabilidad de tamaños entre ellas y la diversidad de factores ambientales que las afectan. Es fundamental asegurar la uniformidad en la distribución de las mismas y la cobertura del objetivo para maximizar la eficiencia biológica del tratamiento (Bayer et al., 2012). Por otro lado, también se debe tener en cuenta la reducción de pérdida de producto al ambiente y a cualquier lugar que no sea el objetivo de aplicación.

Los parámetros de las pulverizaciones que influyen en la calidad de aplicación se pueden definir como, Diámetro Volumétrico Mediano (DV0,5), Densidad de gotas por centímetro cuadrado (gotas $\mathrm{cm}^{-2}$ ) y Cobertura, entre otros (Sarubbi, 2010). La eficiencia también es un parámetro importante de la aplicación de los 
fitosanitarios y se define como la cantidad de volumen de caldo que llega al objetivo en relación al volumen total aplicado. La diferencia entre ellos es el volumen de caldo que se derivó en el proceso (Mur et al., 2017).

Por otro lado, en el último tiempo, los productores y contratistas se han volcado a prácticas con tasas de aplicación reducidas y mayores velocidades de desplazamiento de la máquina pulverizadora, buscando aumentar la capacidad operativa de las máquinas. Sin embargo, los cambios en estos factores y en las condiciones deoperación, asociados a la tecnología de aplicación, pueden influir negativamente en los niveles de depósitos de aspersión y aumentar las pérdidas por deriva principalmente asociadas a gotas medianas y finas (Merani et al., 2019), lo que puede resultar en una menor eficiencia del tratamiento fitosanitario y un mayor impacto ambiental. En este sentido, todas las gotas que no alcancen el objetivo o no queden retenidas en él se conoce como deriva, y constituyen una pérdida del agroquímico aplicado y una consecuente reducción de la eficiencia de la pulverización (Mur et al., 2018). Dicho fenómeno, puede darse por arrastre del producto con las gotas del asperjado o por volatilidad, y este desplazamiento puede quedar dentro del lote o cultivo pulverizado, generando endoderiva (asociadas a gotas de mayor tamaño) o llevar los fitosanitarios fuera del área pulverizada dando como resultado exoderiva (asociadas a gotas de menor tamaño) del producto (Merani et al., 2019).

La técnica de pulverización es uno de los procesos más ineficientes, existe una brecha muy grande entre eficiencia de aplicación y correcto control de la plaga (Alvarez, 2016). Merani et al. (2019) mencionan porcentajes de eficiencia entre 53 y $63 \%$ con diferentes velocidades y tasas de aplicación, y a 0,75 $\mathrm{m}$ de altura sobre el objetivo.

Como se ha mencionado, la eficacia del control en una pulverización agrícola depende de la cantidad de gotas depositadas en los objetivos de interés (hojas, frutos, el suelo, un insecto, un hongo, etc.). La forma de cuantificarlo es evaluando la cobertura y estimando el volumen de aplicación en función del tamaño y número de gotas (Matthews et al., 2014). Por eso, en cada aplicación, es importante conocer la distribución de diámetros de gotas, puesto que determina las características de operación y la eficacia de los dispositivos de pulverización involucrados (Castillo, 2010). Existe un gran interés en caracterizar las aplicaciones y comparar los equipos de pulverización con el fin de mejorar la calidad de aplicación de los plaguicidas; sin embargo, la comparación implica emplear algún tipo de método cuantitativo que permita evaluar la población de gotas, los depósitos o la deriva (Pi Puig, 2019).

La literatura otorga una gran variabilidad de alternativas para evaluar la labor de pulverización a campo que proporcionan información confiable (Gregorio et al., 2011). Una clasificación general de estas metodologías de evaluación, las puede dividir entre las que utilizan colectores artificiales $\mathrm{y}$ las que toman muestras directamente del objetivo.

Los colectores artificiales son elementos como tiras papel absorbente, películas de Mylar o plástico, recipientes de vidrio, papel sensible al agua o el aceite. Dentro de este grupo, la metodología que emplea tarjetas de papel hidrosensible o "Water Sensitive Paper" (WSP) de Sygenta ${ }^{\circledR}$, son consideradas por varios autores (Sayinci et al., 2011; Zhu et al., 2011; Cunha et al., 2012) como la técnica más popular para determinar el espectro de gotas generado por una pulverización, ya que es una técnica relativamente simple de implementar en campo y suministra información confiable sobre el espectro de gotas (CASAFE, 2018).

Dado que sobre la tarjeta hidrosensible se observan manchas y no gotas, hay que considerar el factor de expansión que sufre la gota al chocar con la superficie de la tarjeta, si se quiere conocer el diámetro original de la misma (Domper et al., 2014).

Existen otros papeles, como el kromecote, que se pueden utilizar para la cuantificación de pulverizaciones pero requieren la adición de colorantes para su visualización, ya que no modifican su color por si solos (Ferguson et al., 2016). Estos papeles poseen diferentes factores de expansión de la gota, por lo que se hallarían diferencias en la evaluación al utilizar las herramientas diseñadas para tarjetas hidrosensibles.

Mediante la utilización de lupa puede realizarse el recuento de las manchas o simplemente comparando con cartillas que poseen tarjetas analizadas; sin embargo, los parámetros referidos a diámetros medios (volumétrico y numérico) y consecuentemente volumen de aplicación, no podrían ser determinados. Por otro lado, gotas con diámetro inferior a $50 \mu \mathrm{m}$ (micrones) no serían detectables por esta metodología (Leiva y Araujo, 2009: Cunha et al., 2012; Cunha et al., 2013).

En razón de lo expuesto, y haciendo uso de la tecnología disponible en análisis de imágenes y cálculo computarizado, existen programas diseñados especialmente para cuantificar y caracterizar una aspersión a partir de las improntas generadas por las gotas que alcanzan la tarjeta hidrosensible. Del análisis de la misma, 
se pueden obtener los siguientes parámetros: impactos $\mathrm{cm}^{-2}$, Diámetro Volumétrico Mediano, Cobertura, Diámetro Numérico Mediano, Amplitud Relativa, Factor de Dispersión, DV 0,1, DV 0,9, Eficiencia (Lauric et al., 2016)

Entre los programas más utilizados, se pueden mencionar: CIR 1.5, StainMaster 1.0.8, ambos desarrollados en Argentina; AgroScan, Gotas y e-Sprinkle, desarrollados en Brasil; DropletScan y StainAnalysis, desarrollados en USA y Canadá, respectivamente. Los mismos se desarrollaron con el fin de acelerar y proporcionar una mayor precisión en la aplicación de agroquímicos (Cunha et al., 2013).

La metodología de análisis de las tarjetas hidrosensibles antes descripta es utilizada a nivel productivo. A nivel experimental, se pueden utilizar trazantes fluorimétricos o colorimétricos que permiten, por medio de análisis químico de laboratorio, la determinación precisa de la cantidad de producto efectivamente depositado sobre el objeto de aplicación (Villalba et al., 2011). Un trazador puede ser el ingrediente activo en una mezcla de plaguicidas o un químico seleccionado para imitar al plaguicida, sin que influya en la tensión superficial del agua (Palladini et al., 2005). Un colorante muy empleado es el azul brillante por poseer buena estabilidad a la luz solar (Ferguson et al., 2016).

En función de lo expuesto, el trabajo tiene por objetivo comparar diferentes colectores y programas de evaluación de espectro de gotas, a partir de aplicaciones con diferentes diseños de boquillas.

\section{MATERIALES Y MÉTODOS}

El ensayo se llevó a cabo en la Estación Experimental Julio Hirschhörn, perteneciente a la Facultad de Ciencias Agrarias y Forestales de la Universidad Nacional de La Plata, Buenos Aires, Argentina $\left(34^{\circ} 35 \mathrm{~S}, 57^{\circ} 57 \mathrm{O}\right)$, dentro de un galpón, en condiciones semicontroladas, sin viento $y$ sobre terreno uniforme. Se utilizó una máquina pulverizadora montada, Hatsuta HS400, con bomba de pistones y capacidad de 400 L, botalón con portaboquillas múltiples para tres boquillas, separados 0,52 m entre sí. La pulverizadora fue montada en un tractor New Holland TT.55.

Se seleccionaron dos boquillas con características de pulverización diferentes, pero con el mismo caudal por minuto (Tabla 1). Se trabajó en iguales condiciones de presión y velocidad de desplazamiento a fin de obtener idénticas tasas de aplicación ( $\left.\mathrm{L} \mathrm{ha}^{-1}\right)$.

Las mismas fueron evaluadas a 2,5 bares $(250$ kpa) de presión y a una velocidad de trabajo de $17 \mathrm{~km} \mathrm{~h}^{-1}$. El caudal de las pastillas se ajustó para erogar la dosis deseada $\left(50 \mathrm{~L} \mathrm{ha}^{-1}\right)$ por medio de la variación de la presión de trabajo, en el rango estipulado por tablas del fabricante.

El botalón circuló por encima de los objetivos de pulverización a una altura de $0,75 \mathrm{~m}$.

Durante la realización del ensayo se efectuaron mediciones de las condiciones ambientales mediante una estación meteorológica Kestrel modelo 3500DT. La humedad relativa se mantuvo en valores del 89 al 100\%, la velocidad del viento varió de 0 a $0,5 \mathrm{~m} \mathrm{seg}^{-1}$, la temperatura se halló entre 14,2 y $15,4{ }^{\circ} \mathrm{C}$ y la presión atmosférica fue de 1018 hpa (1,004 atm).

\section{Tarjetas hidrosensibles y fotográficas}

Los objetivos de pulverización utilizados fueron tarjetas hidrosensibles Syngenta ${ }^{\circledR}(0,026$ $\mathrm{m} \times 0,076 \mathrm{~m}$, color amarillo), papel blanco fotográfico tipo glossy de $230 \mathrm{~g} \mathrm{~m}^{-2}(0,026 \mathrm{~m}$ x $0,076 \mathrm{~m}$, color blanco) y papel Whatman 40 $(0,026 \mathrm{~m} \times 0,076 \mathrm{~m})$ para la recolección del trazador. Los mismos fueron colocados sobre las diferentes caras de dos primas rectangulares de distintas dimensiones. Estos últimos estuvieron ubicados sobre pequeños bancos de madera de 0,5 $\mathrm{m}$ de largo por $0,15 \mathrm{~m}$ de ancho, tal como se observa en la Fig. 1. El papel fotográfico glossy fue seleccionado, ya que es el más utilizado en la impresión fotográfica con impresoras de chorro de tinta por su gran nitidez. El mismo posee una capa de revestimiento superior que incluye una alúmina pirógena (pirogénica) con polivinilpirrolidona como aglutinante y capas de revestimiento de base que comprenden carbonato de calcio precipitado y pigmentos de arcilla calcinada con aglutinante de acetato de polivinilo. Esto otorga al papel brillo y las hojas impresas no son pegajosas ni ásperas al tacto, se secan rápido

Tabla 1. Características de las boquillas.

Table 1. Characteristics of the nozzles.

\begin{tabular}{lcccccc}
\hline Boquillas & Ángulo & $\begin{array}{c}\text { Caudal } \\
\left(\mathbf{L ~ m i n}^{-1}\right)\end{array}$ & Tipo & Marca & $\begin{array}{c}\text { Altura } \\
\text { botalón (m) }\end{array}$ & $\begin{array}{c}\text { Tamaño de } \\
\text { gota *** }\end{array}$ \\
\hline XR11002VP & $110^{\circ}$ & 0,79 & Abanico Plano (AP) & Teejet & 0,75 & $\mathrm{~F}$ \\
TXA8002VK & $80^{\circ}$ & 0,79 & Cono Hueco (CH) & Teejet & 0,75 & $\mathrm{~F}$ \\
\hline
\end{tabular}

*** Norma ASAE S572 (ASAE, 2009). 


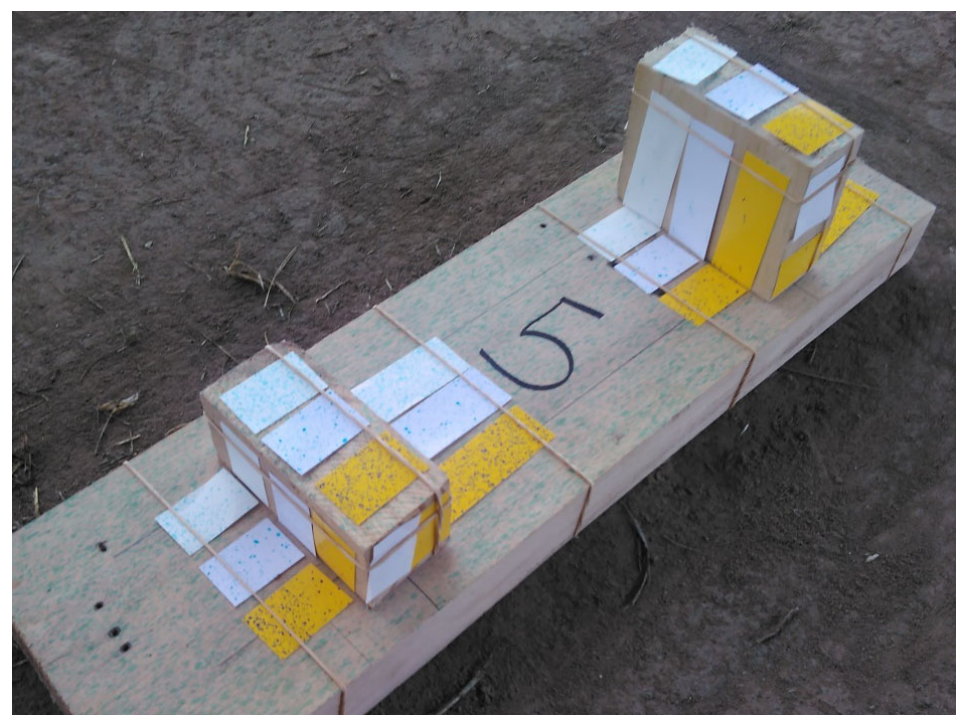

Fig. 1. Banco de prueba donde se observan los prismas con los distintos colectores (papel hidrosensible y paper fotográfico) colocados en las diferentes posiciones.

Fig. 1. Test bench showing the prisms with both collector types (water-sensitive paper and photographic paper) placed in different positions.

(menos de 60 segundos) y los puntos de tinta son redondos, con límites definidos y densidad de color uniforme (Monie, 2002).

Se colocaron 6 bancos de prueba, distribuidos en 2 hileras con 3 repeticiones cada una. Cada repetición estuvo distanciada a $2 \mathrm{~m}$ en la dirección de avance y a 0,75 en sentido transversal, respecto a la otra hilera. Esto permitió obtener objetivos que sean coincidentes con la boquilla de pulverización y objetivos que se hallen entre boquillas, y de esta manera, absorber con las repeticiones la existencia de desuniformidad a lo largo del botalón.

Luego del pasaje de la máquina, se recogieron las tarjetas hidrosensibles y se colocaron en ambiente libre de humedad hasta su análisis. En gabinete, se realizó el procesamiento de las mismas junto con las tarjetas fotográficas, el mismo consiste en dos etapas, una primera digitalización de las tarjetas por medio de scanner Cannon LIDE90, y luego, una segunda etapa, donde son procesadas con los programas CIR 1.5 (Editor) y Gotas PC, obteniendo los siguientes parámetros que constituyeron en parte, las variables de respuesta:

- Densidad de impactos $\left(\mathrm{N}^{\circ}\right.$ de improntas $\left.\mathrm{cm}^{-2}\right)$.

- Diámetro Volumétrico Mediano (DV0,5 o DVM, $\mu \mathrm{m})$, diámetro de gota que divide el volumen pulverizado a la mitad.

- Eficiencia (\%), relación entre lo recogido en el objetivo y lo aplicado.

\section{Trazador}

El trazador utilizado fue colorante alimenticio azul brillante (FD\&C $\mathrm{N}^{\circ}-1$ ), ya que el mismo mantiene la tensión superficial de soluciones acuosas y cumple con las características necesarias de no degradarse con la luz y el tiempo (Palladini et al., 2005). La concentración utilizada fue de 0,1 $\%\left(1 \mathrm{~g} \mathrm{~L}^{-1}\right)$.

Los objetivos de recolección consistieron en hojas de papel Whatman del mismo tamaño que las tarjetas hidrosensibles Syngenta ${ }^{\circledR}$ que luego del pasaje de la máquina pulverizadora, se dejaron secar para su posterior manipuleo. Esta metodología permite, a través de la cuantificación de la concentración de colorante en el objetivo, la determinación precisa de la eficiencia de aplicación de la pulverización.

En laboratorio, se realizó la extracción del trazador de las tarjetas de papel de filtro por medio de lavado con $20 \mathrm{~mL}$ de agua destilada dentro de tubos Falcón de $50 \mathrm{ml}$, con sumersión del papel y agitación vaivén del tubo en posición horizontal (180 golpes por minuto) durante 1 minuto. De esta manera se aseguró la correcta extracción y recuperación del colorante, que luego fue transvasado a tubos de ensayo para su posterior cuantificación.

La cuantificación por colorimetría con el espectrofotómetro Metrolab 325, consistió en una medición del extracto logrado con una longitud de onda de $630 \mathrm{~nm}$. Los valores de absorbancia obtenidos fueron contrastados con una curva de 
calibración construida a partir de concentraciones conocidas que presentó una ecuación $y=7,4386 x$ - 0,05, con R²: 0,9996 (Palladini et al., 2005).

Los valores de trazador se expresan como porcentaje de eficiencia y se construyen a partir de las concentraciones de trazador obtenidas en las tarjetas en relación a la concentración final del caldo aplicado, lo que da como resultado el porcentaje de producto recuperado en las tarjetas.

Los 6 bancos colocados en cada pasada de pulverización, con todas las tarjetas objetivos en sus respectivas posiciones, funcionaron como repeticiones para cada una de las posiciones.

Todos los datos obtenidos fueron volcados en planillas Excel y procesados estadísticamente mediante el software Infostat ${ }^{\circledR}$ (Versión 2017.1.2). Se realizaron análisis de residuos de los valores $\mathrm{y}$, sobre estos, las pruebas de normalidad por gráficos de cuantiles teóricos (Q-Q plot), el contraste de hipótesis de Shapiro-Wilk (Shapiro y Wilk, 1965) y las pruebas de independencia y homocedasticidad. La totalidad de las variables analizadas no cumplieron con el principio de normalidad, por lo que se efectuaron las transformaciones en acuerdo con el tipo de variable, logaritmo (ln y $\log 10)$, raíz cuadrada (SQRT) y potencias $\left(\mathrm{x}^{2} \mathrm{y} \mathrm{x}^{3}\right)$; sin embargo, las transformaciones no permitieron alcanzar los requisitos necesarios para efectuar los análisis de la varianza. En función de lo mencionado, se decidió realizar un análisis de la varianza no paramétrico, por el método de la prueba de $\mathrm{H}$ de Kruskal Wallis, y comparación de medias por el test basado en ranks de Kruskal Wallis, con una significancia de $5 \%(p \leq 0,05)$. El estadístico de la prueba $\mathrm{H}$ se basa en la suma de los rangos (ranks) asignados a las observaciones dentro de cada tratamiento. Su distribución exacta es obtenida a partir de la consideración de todas las configuraciones posibles de los rangos (ranks) de $\mathrm{N}$ observaciones en $\alpha$ grupos de $\mathrm{n}_{\text {i }}$ observaciones cada uno. Si existen diferencias significativas entre los grupos, puede realizarse una prueba post hoc de a pares. InfoStat permite solicitar comparaciones de a pares entre las medias de los rangos (ranks) de tratamientos y/o contrastes entre medias de los rangos (ranks) de tratamientos. El procedimiento usado para juzgar la significancia de las comparaciones múltiples y los contrastes postulados es el descripto por Conover (1999). Si bien las comparaciones entre tratamientos se realizan a través de las diferencias entre las medias de los rangos (ranks), InfoStat permite, también, visualizar las diferencias entre tratamientos a nivel de medias y medianas de los valores originales de las variables (Balzarini et al., 2008; Di Rienzo et al., 2017).

\section{RESULTADOS Y DISCUSIÓN}

Los resultados obtenidos entre los diferentes colectores para el parámetro densidad de impactos, se hayan fuertemente influenciado por el programa utilizado para el análisis de los mismos. En la Fig. 2 se puede observar que ambos software contabilizaron mayor cantidad de impactos cuando se empleó como colector el papel hidrosensible (tarjetas amarillas) solo con diferencias significativas en CIR1.5. Esto puede estar dado por un mejor desempeño por parte de los programas cuando los colectores presentan mayor contrataste entre el color de fondo y el de la gota. Al respecto, Garcia et al. (2004) mostraron que el análisis realizado por diferentes programas de computadora para cuantificar y calificar el proceso de pulverización, mostró resultados muy diferentes en comparación con los métodos tradicionales de análisis. Los autores atribuyeron los errores, principalmente, a los filtros utilizados para separar el color de fondo del papel (amarillo) del color de las gotas (azul). El análisis de imágenes escaneadas de papel amarillo con manchas azules causadas por las gotas, parece simple, pero los autores han identificado alrededor de 90 tonos de colores entre amarillo y azul. La dificultad aumenta, para gotas cuyo tamaño es del orden de micras. En este sentido, Hoffmann et al. (2005) sostienen que hay limitaciones técnicas en las mediciones de gotas sobre papeles hidrosensibles cuando estas son menores a $50 \mu \mathrm{m}$, porque no hay volumen de líquido suficiente para demarcar visiblemente el papel. Los colectores de papel fotográfico (blanco) no presentaron tanto contraste con el trazador azul brillante como los colectores hidrosensibles (Fig. 3), lo que pudo ocasionar que los programas tuviesen dificultades en detectar las gotas más pequeñas, ya que las mismas poseen poca cantidad de trazador colorimétrico, dificultando que el programa pueda separarlas del fondo. Esto podría mejorarse con el aumento de la concentración de trazador en el caldo.

El efecto mencionado anteriormente, solo es notorio con el programa CIR1.5 que detecta gotas más pequeñas. En cambio, el programa GotasPC no presenta diferencias significativas entre colectores, probablemente porque no detecta estas gotas más pequeñas que con el CIR1.5 sobre papel fotográfico se pierden por déficit de trazador.

Por otro lado, existen diferencias estadísticamente significativas entre programas empleando el mismo colector. El software CIR1.5 cuantificó mayor número de impactos con respecto al Gotas-PC tanto con colectores artificiales hidrosensibles (amarillos) como fotográficos 


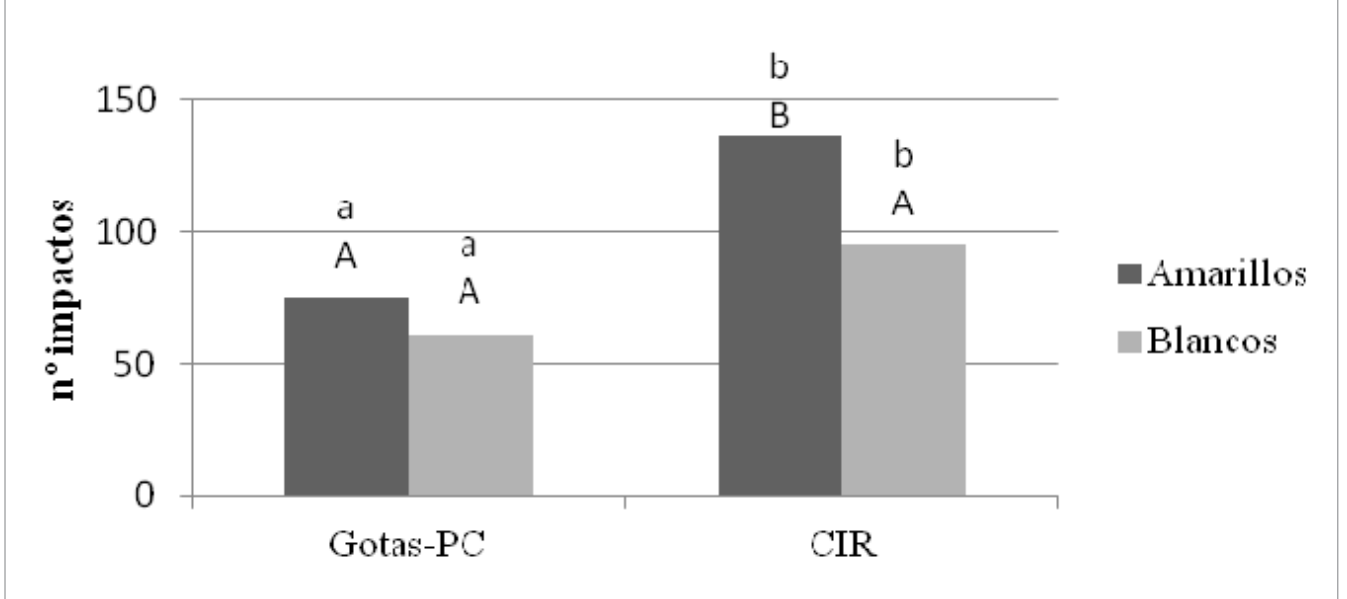

Fig. 2. Densidad de impactos (no impactos $\mathrm{cm}^{-2}$ ) cuantificado por los softwars CIR 1.5 y Gotas-PC, promedio de las dos boquillas. Amarillos: colectores hidrosensibles; Blancos: colectores fotográficos (papel Glossy). Letras minúsculas diferentes indican diferencias significativas $(p \leq 0,05)$ según el test de Ranks de Kruskal Wallis entre programas para cada colector. Letras mayúsculas diferentes indican diferencias significativas $(\mathrm{p} \leq 0,05)$ según el test de Ranks de Kruskal Wallis entre colectores para cada programa.

Fig. 2. Droplet density $\left(\mathrm{n}^{\circ}\right.$ droplet $\left.\mathrm{cm}^{-2}\right)$ quantified by CIR 1.5 and Gotas-PC software programs, average values of the two nozzle types. 'Amarillos': water-sensitive paper; 'Blancos': photographic paper (Glossy paper). Different lowercase letters indicate significant differences $(p \leq 0.05)$ according to the Kruskal Wallis test between software programs for each collector. Different capital letters indicate significant differences $(\mathrm{p} \leq 0.05)$ according to the Kruskal Wallis test between collectors for each software.

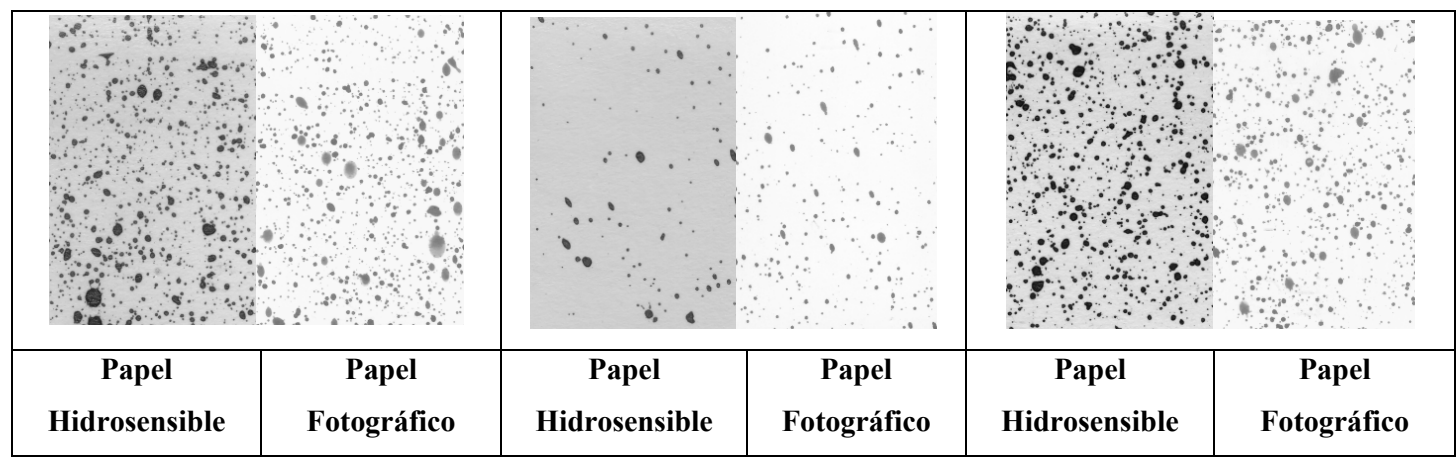

Fig. 3. Imágenes de los colectores (papel hidrosensible y fotográfico), ubicados en la misma posición y escaneados a 1200 dpi.

Fig. 3. Collector images (water-sensitive paper and photographic paper), located in the same position and scanned at $1200 \mathrm{dpi}$.

Glossy (blancos). Estos resultados coinciden con el trabajo realizado por Cunha et al. (2013) en el cual se hallaron diferencias significativas entre los programas Conta Gotas, e-Sprinkle, DepositScan y CIR1.5, siendo este último quien arrojó los mayores valores. Los autores adjudicaron dichas discrepancias, por un lado, a los diferentes factores de corrección que no están especificados, con la excepción de DepositScan, el cual emplea el factor propuesto por Salyani y Fox (1994). Por otro lado, también mencionan la sensibilidad para separar gotas superpuestas como otra fuente de variación en los resultados, basándose en los trabajos de Leiva y Araujo (2009), quienes han encontrado diferencias en la información proporcionada por los diferentes programas de 
conteo y tipificación tanto en la variable cobertura como en las variables de dimensiones de gotas. Dichos autores concluyen en su investigación que el software CIR1.5 fue el que mejor logró el conteo individual de impactos sobre las tarjetas hidrosensibles dada su, ya mencionada, sensibilidad, y por ende alcanzó los valores más altos en número de impactos. La característica de poder individualizar cada una de las gotas es muy importante en tratamientos terrestres donde la cobertura es mayor, y no tanto en los aéreos, donde la cantidad de gotas difícilmente supere las 40 a 45 impactos por $\mathrm{cm}^{-2}$ (Leiva y Araujo, 2009).

Los menores valores arrojados por el software gotas pueden deberse, al no contar con la capacidad de separar gotas superpuestas, a que las improntas pequeñas que tocan a las más grandes no se contabilizan y solo se tienen en cuenta las gotas pequeñas que están aisladas; aspecto que fue estudiado por Leiva y Araujo (2009) y Cunha et al. (2012).

Puesto que las recomendaciones para lograr eficacia biológica de un tratamiento con fitosanitarios habitualmente están dadas por la densidad de impactos $\mathrm{cm}^{-2}$, la información brindada por un determinado software debe ser cuidadosamente utilizada.

Al examinar el tamaño de gotas cuantificado a partir del DV0,5 (Fig. 4), puede observarse un cambio en la tendencia de los resultados en comparación a lo evaluado en el parámetro Densidad de impactos. Gotas-PC fue el que arrojó los mayores valores, tanto en colectores hidrosensibles como en papel fotográfico. Esto puede explicarse teniendo en cuenta que Gotas-PC no detecta la superposición de gotas, computando las improntas como una sola gota de mayor tamaño, y tampoco detecta las gotas muy pequeñas. En tanto, CIR1.5 computa dos o más gotas de menor tamaño en la misma impronta con superposiciones, y detecta las improntas de las gotas más pequeñas, por lo tanto es esperable el menor DV0,5, ya que cuanto más pequeñas sean las gotas que pueda leer un programa, menor será su DV0,5. Estos resultados coinciden con el trabajo realizado por Cunha et al. (2013) donde dicho programa fue el que obtuvo menores valores de DV0,5.

Además, es probable que los software utilicen diferentes factores de expansión para determinar el diámetro de la gota a partir de la impronta en las tarjetas. Esto explicaría, en parte, la diferencia encontrada entre colectores, ya que los

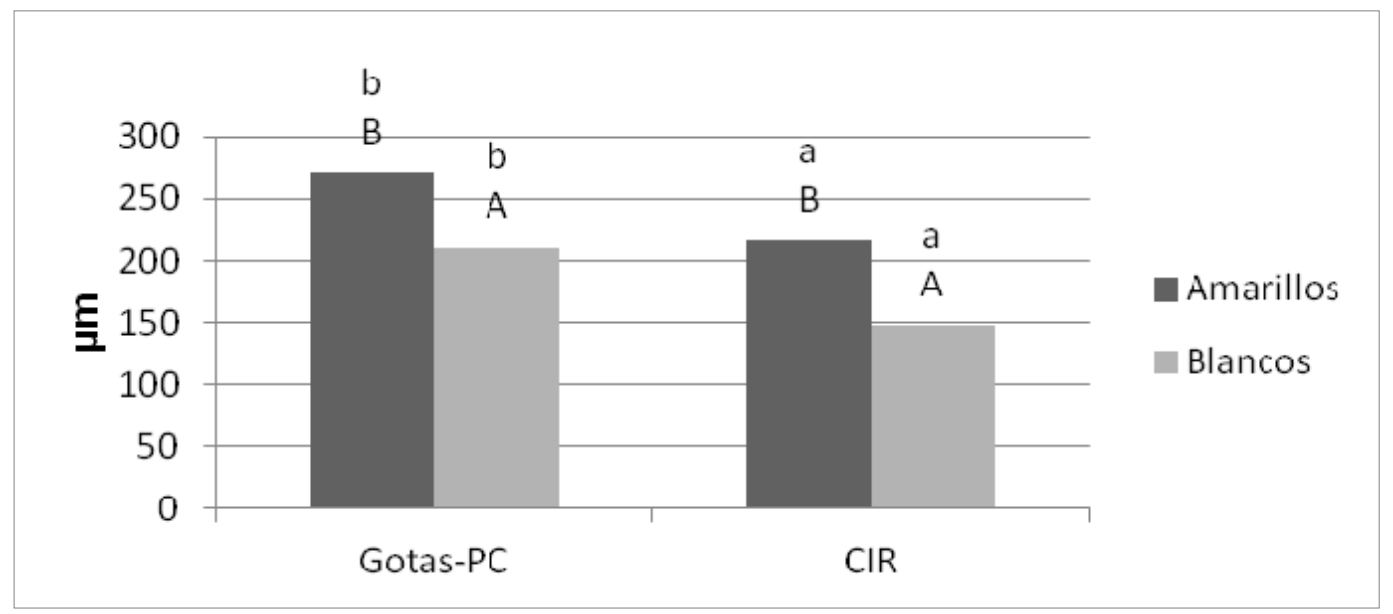

Fig. 4. DV0,5 $(\mu \mathrm{m})$ cuantificado por los software CIR 1.5 y Gotas-PC, promedio de las dos boquillas. Amarillos: colectores hidrosensibles; Blancos: colectores fotográficos (papel Glosy). Letras minúsculas diferentes indican diferencias significativas $(p \leq 0,05)$, según el test de Ranks de Kruskal Wallis entre programas para cada colector. Letras mayúsculas diferentes indican diferencias significativas $(p \leq 0,05)$, según el test de Ranks de Kruskal Wallis entre colectores para cada programa.

Fig. 4. DV0,5 $(\mu \mathrm{m})$ quantified by CIR 1.5 and Gotas-PC software programs, average values of the two nozzle types. 'Amarillos': water-sensitive paper; 'Blancos': photographic paper (Glossy paper). Different lowercase letters indicate significant differences $(p \leq 0.05)$ according to the Kruskal Wallis test between software programs for each collector. Different capital letters indicate significant differences $(\mathrm{p} \leq 0.05)$ according to the Kruskal Wallis test between collectors for each software. 
programas fueron diseñados para trabajar sobre papel hidrosensible donde la gota al impactar se expande, mientras que el papel fotográfico esta específicamente fabricado para reducir al mínimo la expansión de la misma. Estas gotas que no sufren expansión, al ser procesadas por los programas, sufren una reducción del diámetro, arrojando valores menores a la gota real. Baio et al. (2015) compararon las metodologías de uso del papel hidrosensibles y papel fotográfico alternativo contrastando contra mediciones de laser como testigo, destaca que en pastillas $\mathrm{XR}$ con $70 \mathrm{~L}$ $\mathrm{ha}^{-1}$ el papel fotográfico y hidrosensible fueron muy parecidos al laser, siendo el primero el más cercano, en $100 \mathrm{~L} \mathrm{ha}^{-1}$ el papel hidrosensible tuvo un DV0,5 más alto de lo esperado mientras que el fotográfico fue más parecido a los valores de láser. En pastillas inducidas por aire se obtienen valores discordantes. Los autores concluyen que ambos colectores caracterizan igualmente el espectro de gotas y no detectan las gotas muy finas, a diferencia de lo que se encontró en el presente trabajo. Estas discrepancias pueden deberse a dos factores, por un lado, la utilización del CIR1.5 que, a diferencias del Gotas-PC, sí detecta gotas pequeñas; por otro lado, el colorante y las concentraciones utilizadas son muy diferentes, lo que también pudo influir en las diferencias entre colectores. En la Fig. 5 se muestran imágenes de colectores fotográficos e hidrosensibles. En los primeros se observa un halo de menor coloración en el borde de algunas gotas, que no se visualiza en las tarjetas hidrosensibles. Esto puede deberse a la concentración y tipo de trazador que podría generar diferencias en las mediciones de diámetro de las gotas.

En función de lo analizado hasta aquí, si se calibra la concentración de trazador, podría utilizarse papel fotográfico como opción del papel hidrosensible. En parte, esto mejoraría la capacidad de evaluar tamaños de gotas más reales y se eliminaría la necesidad de utilizar coeficientes de expansión en los programas que, por otro lado, según Hoffmann et al. (2005), deberían ser diferentes para cada uno de los tamaños de gotas, lo que dificulta mucho el correcto funcionamiento de los programas.

Es importante tener en cuenta que, aunque existen diferentes alternativas para evaluar tamaños de gotas mucho más precisas como son los sistemas Oxford Laser VisiSizer, Laser diffraction, PDPA y stroboscopic imaging presentadas por Sijs et al. (2021), las mismas solo reflejan los tamaños de gotas que salen de las boquillas en condiciones controladas de laboratorio. Estos valores suelen estar expresados en las tablas de los fabricantes de boquillas, y si bien se toman como referencia en el análisis de los resultados obtenidos en los colectores, las gotas que alcanzan los objetivos suelen estar fuertemente influenciadas por las condiciones ambientales al momento de la aplicación, generando una reducción del tamaño de las mismas y la desaparición de las más pequeñas, lo que afecta los resultados finales de DV0,5, DV0,1 y DV0,9. Debe tenerse en cuenta los valores de referencia a la hora de seleccionar boquillas, pero teniendo presente que los resultados finales sobre el objetivo serán diferentes y deberán ser medidos por algún sistema de evaluación a campo.

En cuanto al parámetro de eficiencia de aplicación que construyen y arrojan los programas de evaluación de tarjetas, si bien es muy poco robusto, es muy utilizado a campo para evaluar la llegada del producto al objetivo. La metodología de trazador aporta robustez al análisis de eficiencia, pero en situaciones productivas es muy difícil de ser utilizado. Es por ello que, la comparación de esta metodología con diferentes programas y colectores ayudaría a seleccionar la combinación más precisa $\mathrm{y}$, de ser necesario, poder ajustar los porcentajes al momento de evaluar eficiencia a campo para que sean los más real y representativo de la situación objetivo. En este sentido, Stoletniy et al. (2014), afirman que la metodología de trazador es de gran precisión, pero su uso es engorroso y requiere de trabajo en laboratorio y equipos complejos.

$\mathrm{Al}$ realizar el análisis estadístico de la variable eficiencia (Fig. 6), se observa que los programas,

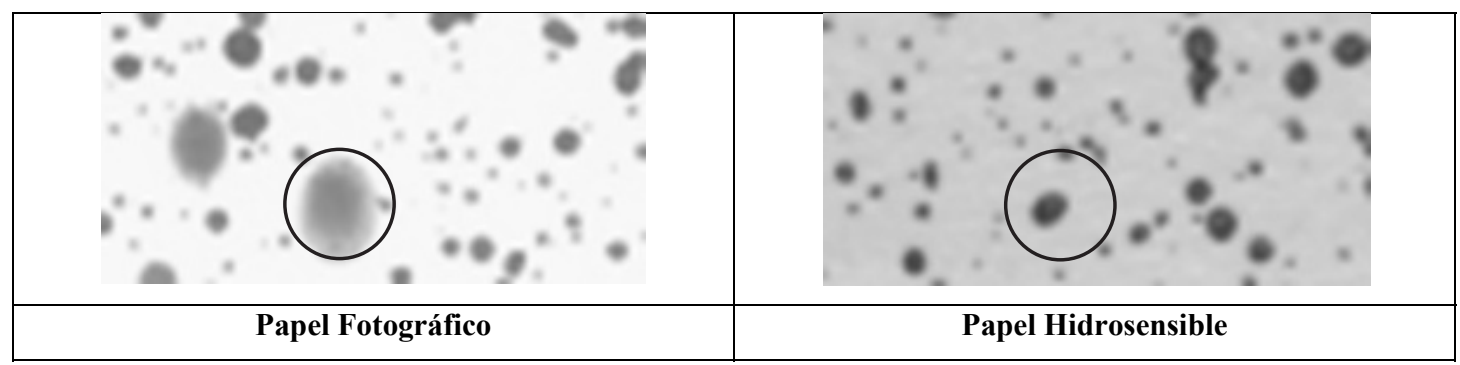

Fig. 5. Detalle de la gota sobre papel fotográfico e hidrosensible ampliado $5 \mathrm{X}$.

Fig. 5. Drop detail on photographic and water-sensitive paper $5 \mathrm{X}$ magnified. 


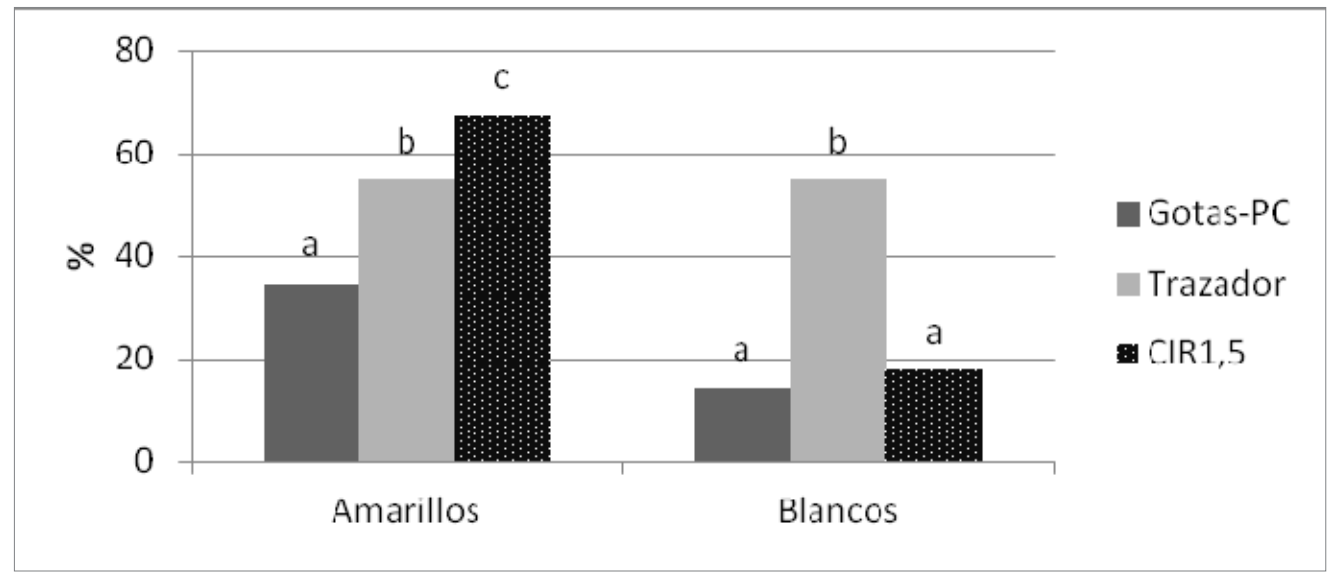

Fig. 6. Eficiencia de aplicación (\%) cuantificada por la metodología de trazador colorimétrico y los software CIR 1.5 y Gotas-PC, sobre colectores Amarillos (papel hidrosensible) y Blancos (papel fotográfico Glosy), promedio de las dos boquillas. Letras diferentes indican diferencias significativas $(\mathrm{p} \leq 0,05)$, según el test de Ranks de Kruskal Wallis.

Fig. 6. Application efficiency (\%) quantified by the colorimetric tracer methodology and CIR $\mathbf{1 . 5}$ and Gotas-PC software programs, average values of the two nozzle types. 'Amarillos': watersensitive paper; 'Blancos': photographic paper (Glossy paper). Different letters indicate significant differences $(\mathrm{p} \leq 0.05)$ according to the Kruskal Wallis test.

independientemente del tipo de colector, difieren significativamente de lo arrojado por el trazador colorimétrico.

Las evaluaciones con colectores blancos mostraron, para ambos programas, porcentajes muy bajos en comparación con el trazador. Esto se puede explicar a partir de la construcción del valor de eficiencia que realizan los programas, los cuales utilizan los parámetros de densidad de impactos y tamaño de gotas para su estimación. Dado que los valores de dichas variables fueron menores en los colectores fotográficos respecto a las tarjetas hidrosensibles, la estimación de los volúmenes se vio sumamente disminuida. Del mismo modo, la ausencia de diferencias estadísticas entre programas para los colectores blancos, se debe a la compensación entre variables intervinientes para el cálculo de este parámetro. Es decir, el Gotas-PC arrojó menor densidad de impactos, pero de mayor tamaño; en tanto, el CIR 1.5 detectó mayor número de impactos, pero el tamaño de la población fue menor. Estos resultados demuestran una vez más que el software Gotas-PC no detecta las gotas más pequeñas.

Cuando se utilizaron colectores hidrosensibles, CIR1.5 presentó valores significativamente superiores a los de Gotas-PC, lo que se explica por la mayor cuantificación de impactos, cercano al doble, que los indicados por Gotas-PC. Si bien el Gotas-PC determina mayor DV0,5 que el CIR1.5, no compensa la gran cantidad de impactos precisados por el CIR1.5. Asimismo, CIR1.5 sobreestima la eficiencia de aplicación en relación al trazador colorimétrico, mientras que Gotas-PC la subestima. Esto puede deberse a que no todos los programas calculan el área de la gota de la misma forma, ya que la gota no es perfectamente circular, y utilizan diferentes fórmulas para poder llegar al área de cada una (Zhu et al., 2011), lo que puede generar errores de estimación y discordancias entre programas.

Respecto al comportamiento de las boquillas, en la Tabla 2 se muestran los resultados de las variables particionadas, según colector $\mathrm{y}$ programa. La boquilla AP (XR11002VP) presentó diferencias significativas en los colectores hidrosensibles entre programas, en todos los parámetros; mientras que, con colectores fotográficos, no llegan a visualizarse diferencias significativas en Eficiencia y Cobertura, pero sí en Densidad de impactos y DV0,5. Al mismo tiempo se visualiza que el parámetro Densidad de Impactos para el programa Gotas-PC es el único que no tiene diferencias significativas entre colectores.

En la boquilla $\mathrm{CH}$ (TXA8002VK) no se visualizan diferencias significativas entre programas para ninguno de los dos colectores en Eficiencia y Cobertura, pero sí se observan en Densidad de impactos y DV0,5. En función de esto, se podría suponer que las determinaciones 
Tabla 2. Valores para todos los parámetros evaluados particionados por boquilla, según programa y colector. AP: XR11002VP; CH: TXA8002VK; Amarillos: colectores hidrosensibles; Blancos: colectores fotográficos (papel Glossy). Letras diferentes indican diferencias significativas $(p \leq 0,05)$ según el test de Ranks de Kruskal Wallis entre programas y colectores: para cada boquilla.

Table 2. Values for all evaluated parameters partitioned by nozzle type according to software and collector AP: XR11002VP; CC: TXA8002VK; 'Amarillos: water-sensitive paper; 'Blancos': photographic paper (Glossy paper). Different letters indicate significant differences $(p \leq 0.05)$ according to the Kruskal Wallis test between software programs and collectors for each nozzle.

\begin{tabular}{clccccc}
\hline Pastillas & Colectores & Programas & $\begin{array}{c}\text { Densidad de } \\
\text { impactos }\end{array}$ & $\begin{array}{c}\text { DV0,5 } \\
(\boldsymbol{\mu m})\end{array}$ & $\begin{array}{c}\text { Cobertura } \\
(\mathbf{\%})\end{array}$ & $\begin{array}{c}\text { Eficiencia } \\
(\mathbf{\%})\end{array}$ \\
\hline AP & Amarillos & Gotas-PC & $71,08 \mathrm{ab}$ & $288,59 \mathrm{c}$ & $5,48 \mathrm{~b}$ & $25,44 \mathrm{~b}$ \\
& & CIR1,5 & $\begin{array}{c}119,21 \mathrm{c} \\
252,20 \mathrm{~b}\end{array}$ & $7,39 \mathrm{c}$ & $52,70 \mathrm{c}$ \\
& \multirow{2}{*}{ Blancos } & Gotas-PC & $63,40 \mathrm{a}$ & $240,90 \mathrm{~b}$ & $2,73 \mathrm{a}$ & $10,95 \mathrm{a}$ \\
& & CIR1,5 & $85,77 \mathrm{~b}$ & $166,46 \mathrm{a}$ & $2,59 \mathrm{a}$ & $13,62 \mathrm{ab}$ \\
$\mathrm{CH}$ & \multirow{2}{*}{ Amarillos } & Gotas-PC & $79,52 \mathrm{a}$ & $252,70 \mathrm{c}$ & $5,97 \mathrm{~b}$ & $28,14 \mathrm{~b}$ \\
& & CIR1,5 & $150,82 \mathrm{c}$ & $182,82 \mathrm{~b}$ & $6,58 \mathrm{~b}$ & $38,85 \mathrm{~b}$ \\
& \multirow{2}{*}{ Blancos } & Gotas-PC & $59,73 \mathrm{a}$ & $180,02 \mathrm{~b}$ & $1,92 \mathrm{a}$ & $8,13 \mathrm{a}$ \\
& & CIR1,5 & $104,44 \mathrm{~b}$ & $131,17 \mathrm{a}$ & $2,45 \mathrm{a}$ & $11,34 \mathrm{a}$ \\
\hline
\end{tabular}

de los parámetros Cobertura y Eficiencia se encuentran muy influenciadas por la interacción entre el tamaño de las improntas y la densidad de impactos. Si la reducción de tamaños es muy marcada, los cálculos de Eficiencia y Cobertura entre programas se equiparán como ocurre con los colectores fotográficos; del mismo modo, cuando el tamaño de la gota es pequeño, la expansión de las mismas es menor, como sucede con los colectores hidrosensibles.

\section{CONCLUSIONES}

El programa CIR 1.5 es el más sensible al momento de analizar las gotas sobre los colectores, y es capaz de distinguir mayor número de gotas y de menor tamaño sobre ambos colectores.

Los colectores de papel fotográfico presentan improntas más pequeñas que arrojan valores de DV0,5 y Eficiencia menores.

Para aplicaciones con gotas de DV0,5 pequeño, los valores de Eficiencia evaluados por ambos programas son similares.

La densidad de impactos no presenta diferencias entre colectores para el programa Gotas-PC, pero si para CIR1.5.

Al utilizar colectores hidrosensibles, la eficiencia es sobrestimada con el programa CIR1.5 y subestimada al usar el programa Gotas-PC. Con colectores fotográficos, ambos programas subestiman.

\section{LITERATURA CITADA}

Alvarez, I. J. M. 2016. Manejo y Uso eficiente del tamaño, uniformidad y distribución de la gota de pulverización en cultivo de soja ( Glycine max L. Merrill ) Universidad Nacional Del Nordeste Facultad De Ciencias Agrarias Maestria En Producción Vegetal. http://repositorio.unne.edu.ar/bitstream/ handle/123456789/478/RIUNNE_TM_ Álvarez_I.pdf?sequence $=1$ \&isAllowed=y

Baio, F. H. R., I. M. Scarpin, e E. E. da Silva. 2015. PAPEL hidrossensível e alternativo fotográfico em ensaios de deposição de gotas. Brazilian Journal of Biosystems Engineering 9,4: 339-347.

Balzarini, M. G., Gonzalez, L., Tablada, M., Casanoves, F., Di Rienzo, J. A., \& Robledo, C. W. (2008). InfoStat. Infostat. Manual del Usuario (Editorial Brujas). Córdoba, Argentina. 336pp.

Bayer, T., A. Arrué, I. F. Dressler da Costa, G. Lenz, B. G. Sari, M. Pizzuti Pes, e C. Cezar. 2012. Aplicação aérea de fungicidas na cultura do arroz irrigado com diferentes bicos de pulverização Aerial fungicide application on irrigated lowland rice with varying spraying nozzles. Ciência Rural 42,12: 2185-2191.

Bernardos, J., y M. E. Zaccagnini. 2011. El uso de insecticidas en cultivos agrícolas y su riesgo potencial para las aves en la Región Pampeana. El Hornero 26,1: 55-64. http://www.scielo.org. ar/scielo.php?script=sci_arttext\&pid=S007334072011000100005\&lng=es\&nrm=iso\&tlng=es 
Carriquiborde, P. 2010. Toxicidad de Glifosato en Peces Autóctonos: Estudios de Laboratorio y Campo. In M. Camino \& V. Aparicio (Eds.), Taller Aspectos Ambientales del Uso de Glifosato (Ediciones, pp. 53-64). https://www.researchgate.net/ publication/288254029\%0AToxicidad

CASAFE. 2018. Uso de Tarjetas Hidrosensibles. https://www.casafe.org/uso-de-tarjetashidrosensibles/

Castillo, B. 2010. Tecnología de aplicación de agroquímicos (J. C. (coordinador) Magdalena, B. Castillo Herrán, A. Di Prinzio, I. Homer Bannister, \& J. Villalba (eds.)).

Conover, W.J. (1999). Practical Nonparametric Statistics. John Wiley \& Sons, Inc., New York. $3^{\circ}$ edition Texas Tech University. 584pp.

Cunha, J. P. A. R., A. C. Farnese, and J. J. Olivet. 2013. Computer programs for analysis of droplets sprayed on water sensitive papers. Planta Daninha 31,3: 715-720. https://doi. org/10.1590/s0100-83582013000300023

Cunha, M., C. Carvalho, and A. R. S. Marcal. 2012. Assessing the ability of image processing software to analyse spray quality on watersensitive papers used as artificial targets. Biosystems Engineering 111,1: 11-23. https:// doi.org/10.1016/j.biosystemseng.2011.10.002

Czepak, C., K. C. Albernaz, L. M. Vivan, H. O. Guimarães, e T. Carvalhais. 2013. Primeiro registro de ocorrência de Helicoverpa armigera (Hübner) (Lepidoptera: Noctuidae) no Brasil. Pesquisa Agropecuaria Tropical 43,1: 110-113. https://doi.org/10.1590/S198340632013000100015

Di Rienzo, J.A., Casanoves F., Balzarini M.G., Gonzalez L., Tablada M., Robledo C.W. (2017). InfoStat versión 2017. Grupo InfoStat, FCA, Universidad Nacional de Córdoba, Argentina. Disponible en: URL http://www. infostat.com.ar. Ultimo acceso: Julio 2020.

Domper, G. N., M. Mur, y R. H. Balbuena. 2014. Eficiencia de aplicación de pastillas de pulverización con inducción de aire en el cultivo de soja. Revista de La Facultad de Agronomia 113,2: 202-210.

FAO, y OMS. 2014. Código Internacional de Conducta para la Gestión de Plaguicidas. https://doi.org/13604S/1/12.14

Ferguson, J. C., R. G. Chechetto, C. C. O'Donnell, B. K. Fritz, W. C. Hoffmann, C. E. Coleman, et al. 2016. Assessing a novel smartphone application - SnapCard, compared to five imaging systems to quantify droplet deposition on artificial collectors. Computers and Electronics in Agriculture 128: 193-198. https://doi.org/10.1016/j.compag.2016.08.022
Garcia, L. C., C. G. Raetano, A. Justino, e C. Puríssimo. 2004. Dessecação da aveia-preta (Avena strigosa Schreb) com herbicida de contato, em presença ou não de assistência de ar junto à barra do pulverizador, em diferentes volumes de calda. Engenharia Agrícola 24,3: 758-763. https://doi. org/10.1590/S0100-69162004000300029

Gregorio, E., F. Solanelles, F. Rocadenbosch, J. R. Rosell, and R. Sanz. 2011. Airborne spray drift measurement using passive collectors and lidar systems. Remote Sensing for Agriculture, Ecosystems, and Hydrology XIII 8174: 12. https://doi.org/10.1117/12.903723

Hoffmann, W. C., and A. J. Hewitt. 2005. Comparison of three imaging systems for water-sensitive papers. ASABE American Society of Agricultural and Biological Engineers 21,6: 961-964.

ISAAA. 2017. Argentina. In Global Status of Commercialized Biotech/GM Crops in 2017.

Lauric, A., G. De Leo, C. Carbonell, M. Vigna, y D. Leiva. 2016. Ensayo exploratorio utilización de tarjetas hidrosensibles para evaluar el efecto de la presión sobre la calidad de aplicación. INTA 1.

Leiva, P. D., y E. C. Araujo. 2009. Comparación de programas de computación para recuento y tipificación de impactos de aspersión sobre tarjetas sensibles. Pergamino: INTA 7.

Matthews, G. A., R. Bateman, and P. Miller. 2014. Pesticide Application Methods. John Wiley \& Sons, Ltd, West Sussex, UK; Oxford, UK 111 Hoboken, USA

Merani, V. H., M. Mur, F. Ramirez, M. J. Ponce, F. Guilino, y T. C. Palancar. 2019. Efecto de variables operativas sobre la calidad de aplicación y la deriva en la pulverización de agroquímicos. AgriScientia 36,2: 45-55. https://doi.org/10.31047/1668.298x.v36. n2.19093

Monie, S. A. 2002. Patent Application Publication ( 10 ) Pub . No .: US 2002 / 0124603 A1: Vol. US 2002/01.

Mur, M., M. Ponce, J. Vázquez, F. Guilino, V. Merani, T. Palancar, y R. Balbuena. 2018. Aplicación de agroquímicos en cultivos de soja (Glycine max L Merr). Evaluación del efecto de diferentes técnicas sobre la eficiencia de distribución. Revista de La Facultad de Agronomía, La Plata 117,1: 77-88.

Mur, M., M. Ponce, J. M. Vázquez, F. Guilino, V. Merani, T. Palancar, y R. H. Balbuena. 2017. Control de malezas en pre siembra. Alternativas tecnológicas y riesgos ambientales de la aplicación. Revista de La Facultad de Ciencias Agrarias y Forestales 116,2: 267-277. 
Palladini, L. A., C. G. Raetano, and E. D. Velini. 2005. Choice of tracers for the evaluation of spray deposits. Scientia Agricola 62,5: 440-445. https://doi.org/10.1590/s010390162005000500005

Pi Puig, F. 2019. Pulverizaciones agrícolas: Comparación de metodologías para su evaluación. Tesis de Grado. Facultad de Ciencias Agrarias y Forestales. Universidad Nacional de La Plata. http://sedici.unlp.edu. ar/handle/10915/85777

Piazza, A., S. García, J. Lazovski, M. Valls, L. Bulacio, y D. Méndez. 2011. Guia de uso responsable de agroquimicos. https:// www.toxicologia.org.ar/bibliotecavirtual/ documentos_utilies/guia_de_uso_de_ agroquimicos.pdf

Salyani, M., and R. D. Fox. 1994. Performance of image analysis for assessment of simulated spray droplet distribution. Transactions of the ASAE 37,4: 1083-1089. https://doi.org/ doi: $10.13031 / 2013.28179$

Sarubbi, C. A. S. 2010. Tecnología de aplicación de productos fitosanitarios en equipos pulverizadores terrestres La gota de aspersión y las boquillas pulverizadoras, Capitulo 4, (FAUBA).

Sayinci, B., and S. Bastaban. 2011. Spray distribution uniformity of different types of nozzles and its spray deposition in potato plant. African Journal of Agricultural Research 6,2: 352-362. https://doi. org/10.5897/AJAR10.480

SENASA (Servicio Nacional de Sanidad y Calidad Agroalimentaria). 2020. https:// www.argentina.gob.ar/senasa/programassanitarios/productosveterinariosfitosanitarios-y-fertilizantes/registronacional-de-terapeutica-vegetal. $\% 20$ Consultado\%20en\%20julio\%202020. Última visita Agosto 2021.

Shapiro, S.S. and Wilk, M.B. 1965. An Analysis of variance test for normality (complete samples). Biometrika, 52 (3-4): 591-611. Sijs, R., Kooij, S., Holterman, H. J., van de Zande, J. and D. Bonn. 2021. Drop size measurement techniques for sprays: Comparison of image analysis, phase Doppler particle analysis, and laser diffraction, AIP Advances 11, 015315. https://doi.org/10.1063/5.0018667
Simoniello, M. F., E. C. Kleinsorge, and M. A. Carballo.2010.Biochemicalevaluationonrural workers exposed to pesticides / Evaluación bioquímica de trabajadores rurales expuestos a pesticidas. Medicina 70: 489-498. http:// www.scielo.org.ar/scielo.php?pid=S002576802010000600001\&script=sci_arttext

Stoletniy, I., J. Olivet \& J. Villalba. 2014. Tamaño de gota, volumen de aplicación y uso de adyuvante en la deposición y control de mancha amarilla (Pyrenophora tritici repentis) en trigo. Agrociencia Uruguay Volumen 18- 1: 97-104

Villalba, Andrea. 2009. Resistencia a herbicidas. Glifosato. Ciencia, Docencia y Tecnología, vol. XX, núm. 39, noviembre, 2009, pp. 169186. Universidad Nacional de Entre Ríos. Concepción del Uruguay, Argentina.

Villalba, J., J. J. Olivet, M. E. Cassanello, O. Bentancur, and J. P. Cunha. 2011. Evaluation of deposition of spray nozzles for control of fusarium in wheat. / Evaluación de la deposición de boquillas de aplicación para el control de fusariosis en trigo. Agrociencia (Montevideo) 15,2 PG-69-75: 69-75. email: villalba@fagro.edu.uy NS -

Zarrilli, A. 2020. La Naturaleza puesta en Jaque: La Expansión de la Frontera Agropecuaria en Argentina y su Impacto Socio-Ambiental (1980- 2017). HALAC - Historia Ambiental, Latinoamericana y Caribeña 10,1: 125-149. https://doi.org/https://doi.org/10.32991/22372717.2020v10i1

Zhu, H., M. Salyani, and R. D. Fox. 2011. A portable scanning system for evaluation of spray deposit distribution. Computers and Electronics in Agriculture 76,1: 38-43. https:// doi.org/10.1016/j.compag.2011.01.003 